\title{
Ion acceleration in a solenoid-free plasma expanded by permanent magnets
}

\author{
K. Takahashi, ${ }^{a)}$ K. Oguni, H. Yamada, and T. Fujiwara \\ Department of Electrical and Electronic Engineering, Iwate University, Morioka 020-8551, Japan
}

(Received 3 June 2008; accepted 9 July 2008; published online 1 August 2008)

\begin{abstract}
Ion acceleration is achieved in a low-pressure solenoid-free plasma expanded by permanent magnet arrays. Although a permanent magnet normally forms cusp magnetic fields which prevents plasma diffusion and double layer formation, by employing double concentric arrays of permanent magnets, a constant field area, and a diverging magnetic field can be generated near the outlet of the plasma source. In the source, a rapid potential drop with $4 \mathrm{~cm}$ thickness from $50 \mathrm{~V}$ to $20 \mathrm{~V}$ is generated at the diverging field area for $0.35 \mathrm{mTorr}$ and a supersonic ion beam accelerated through the potential drop is observed in the diffusion chamber. The beam energy can be increased up to over $40 \mathrm{eV}$ with a decrease in gas pressure. (C) 2008 American Institute of Physics. [DOI: 10.1063/1.2965497]
\end{abstract}

Plasma expansion has attracted a great deal of attention because it self-consistently forms nonlinear plasma-potential structures causing electrostatic ion acceleration. The process has been investigated for a long time in connection with space plasmas ${ }^{1}$ and electric propulsion devices. ${ }^{2}$ The plasmapotential structures are divided broadly into two types: One is an electric double layer (DL) due to charge separation phenomena, another is a potential gradient determined by a pressure gradient, i.e., following the Boltzmann's relation. Recent experiments and theories have shown the DL formation and/or the ion acceleration in expanding plasmas. ${ }^{3-10}$ Charles and Boswell have suggested that the new type of electric thruster, named the helicon double layer thruster, can yield a long-lifetime because it does not require ion extraction electrodes. Recent measurements of the electron energy distribution function upstream of the helicon double layer showed that only a single upstream plasma source is required to maintain the formation of the double layer, ${ }^{10,11}$ hence the above-mentioned ion accelerator is considered to be applicable to the thruster.

Helicon wave discharges, i.e., rf-driven plasmas under steady-state magnetic field, are well known to be the efficient plasma sources for high-density plasma productions. In conventional helicon sources, electromagnets are used for generating the steady-state magnetic fields, which consume much electricity, and make the devices large and costly. From the viewpoint of the industrial applications, compact helicon sources using permanent magnets were developed. ${ }^{12-14}$ More recently, Shamrai et al. developed the compact helicon source introducing the permanent magnets for the electric propulsion. ${ }^{15}$ The magnetic fields produced by the permanent magnets are normally strongly nonuniform with reverse-fields, i.e., cusps. They demonstrated that the cusps prevent the plasma diffusion and the ion beam formation. In order to eliminate the cusps near the outlet of the source tube, the magnetic fields produced by the electromagnets (solenoidal coils) are superimposed in their experiments, where the ion beam of energy above $100 \mathrm{eV}$ is detected as a result of optimizing the magnetic field and the spatial profile

\footnotetext{
${ }^{a)}$ Electronic mail: kazunori@iwate-u.ac.jp.
}

of the gas pressure. In addition to the above experiments, Corr et al. demonstrated the ion beam formation by the Boltzmann electric field in geometrically expanding plasmas. ${ }^{16}$

In the present letter, we demonstrate the formations of the rapid potential drop and the subsequent ion beam in the solenoid-free expanding plasmas using only the permanent magnets. A constant field area and a diverging magnetic field without the cusp near the outlet of the plasma source are provided by double concentric arrays of the permanent magnets, where each array consists of eight magnet bars. The rapid potential drop with about $4 \mathrm{~cm}$ thickness is observed only for the case of low pressure less than 2 mTorr, which is not in agreement with the Boltzmann's relation and is guessed to be the DL; then an ion energy distribution function (IEDF) shows supersonic ion beam accelerated by the potential drop. The beam energy increases up to over $40 \mathrm{eV}$ with a decrease in gas pressure, and the potential drop and the ion beam disappears above 2 mTorr.

A schematic diagram of the experimental setup is shown in Fig. 1(a). A cylindrical glass tube of $20 \mathrm{~cm}$ in length and $6.6 \mathrm{~cm}$ in inner diameter (source tube) is attached contiguously to a $26 \mathrm{~cm}$ diam and $30 \mathrm{~cm}$ long grounded stainless steel vacuum chamber (diffusion chamber). The chamber is evacuated to a base pressure of $2 \times 10^{-6}$ Torr by the diffusion/rotary pumping system, and the argon gas is introduced from the source side through a mass flow controller. An argon plasma is excited by a double-turn loop antenna located at $z=-9 \mathrm{~cm}$ and powered from a rf generator of frequency $13.56 \mathrm{MHz}$ and power $250 \mathrm{~W}$, where $z=0$ is defined as the outlet of the source tube. The double concentric arrays of Neodymium Iron Boron $(\mathrm{NdFeB})$ magnets $(10 \mathrm{~cm}$ in length, $1.5 \mathrm{~cm}$ in width, and $0.5 \mathrm{~cm}$ in thickness) arranged as shown in Fig. 1(b) are set around the source tube, where all of the magnets have inward magnetization in the radial direction (in the direction of thickness). Although the single magnet-array consisting of eight magnet bars forms the cusp at the axial center of the bar without a constant field area, the double arrays with different radii of $5.5 \mathrm{~cm}$ and $7 \mathrm{~cm}$ located at different axial positions can produce the constant field area for plasma production and the diverging field area in the downstream region. The calculated and experimentally mea- 
(a)

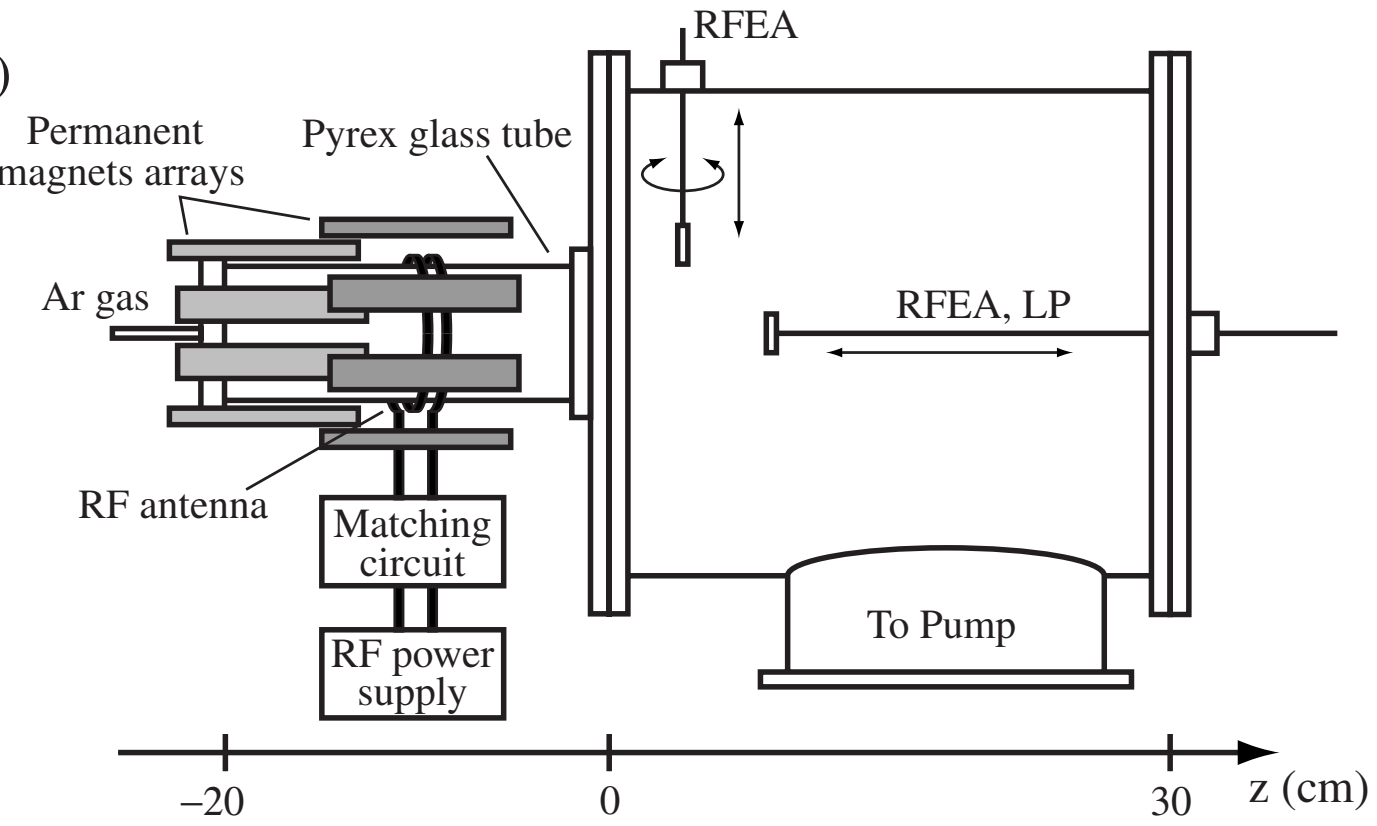

(b)

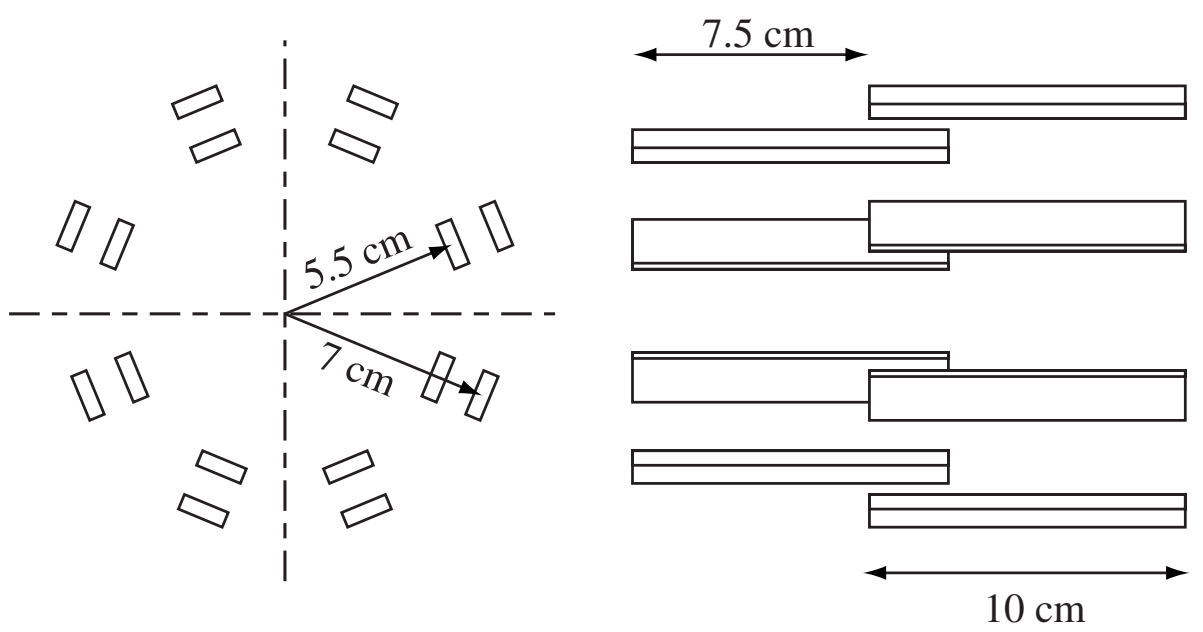

(c)

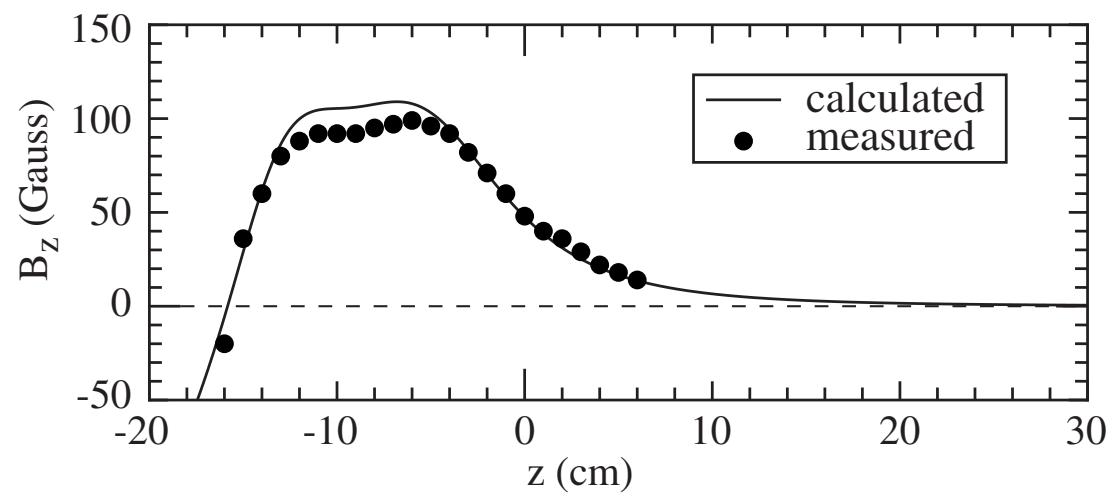

FIG. 1. (a) Schematic diagram of experimental setup. (b) Detailed configuration of the permanent magnets. (c) Axial profile of the calculated (solid line) and experimentally measured (closed circles) axial component $B_{z}$ of the magnetic-field strength produced by the permanent magnets.

sured axial component of the local magnetic-field strength are shown in Fig. 1(c) as solid line and closed circle, respectively. The calculated field strength is in good agreement with the experimentally measured one. It is found that there is no cusps near the outlet of the source tube, although the cusp is formed in the upstream side $(z \simeq-16 \mathrm{~cm})$ of the source.
As shown in Fig. 1(a), a planar Langmuir probe (LP) and two retarding field energy analyzers (RFEAs) are inserted from the downstream side and the side wall of the diffusion chamber for characterizing the plasma density, the plasma potential, and the IEDF. IEDF, i.e., the first derivative curve of a collector current $I_{c}$ of the RFEA depending on the biased voltage $V_{c}$, is directly obtained by a pulsed probe technique 

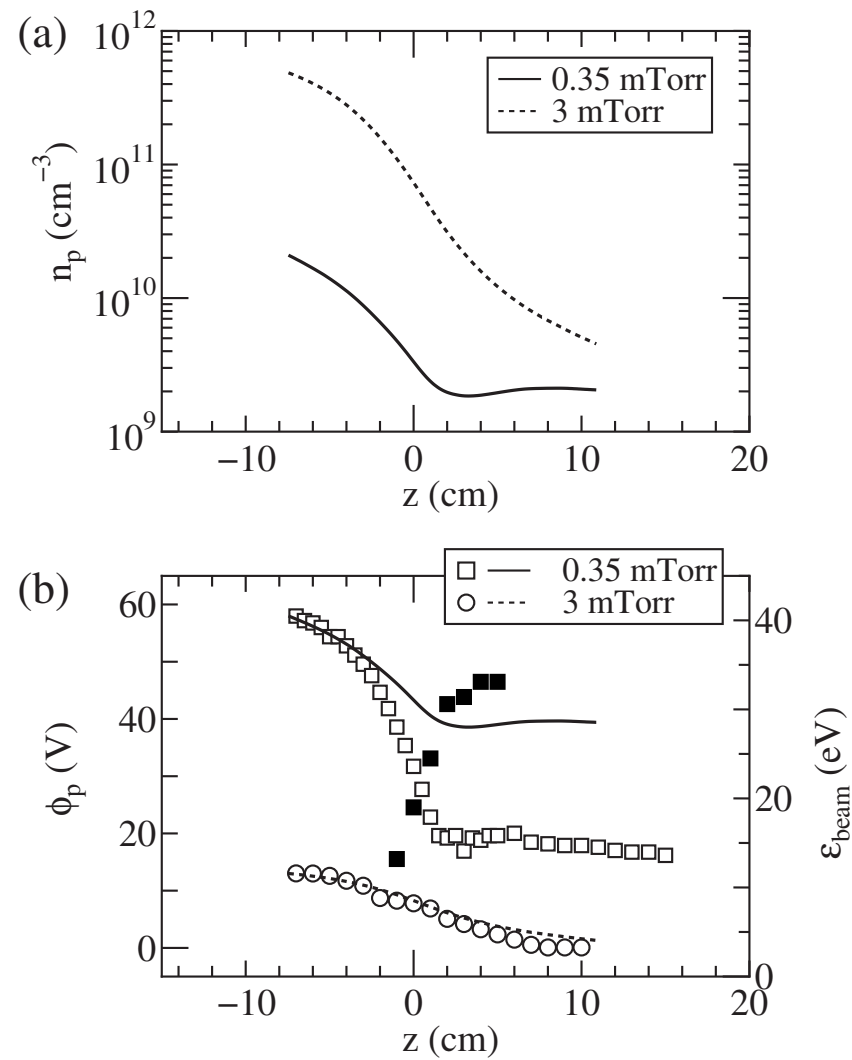

FIG. 2. (a) The measured plasma density $n_{p}$ as a function of axial position $z$ for $0.35 \mathrm{mTorr}$ (solid line) and $3 \mathrm{mTorr}$ (dotted line). (b) The measured plasma potential $\phi_{p}$ for 0.35 mTorr (open squares) and 3 mTorr (open circles). The solid and dotted lines are the plasma potentials estimated using Eq. (1) and the density profiles shown in Fig. 2(a) for 0.35 mTorr and 3 mTorr, respectively. The measured ion beam energy $\varepsilon_{\text {beam }}$ for $0.35 \mathrm{mTorr}$ is also plotted in Fig. 2(b) as a closed square.

using a differentiator reported in previous experiments. ${ }^{11,17}$

Figure 2(a) shows the axial profiles of the plasma density $n_{p}$ estimated from the ion saturation current of the LP for gas pressure of $0.35 \mathrm{mTorr}$ (solid line) and $3 \mathrm{mTorr}$ (dotted line). Here, the LP is transferred by a servomotor and the spatial profile of the ion current can be contiguously obtained as shown in Fig. 2(a). For 3 mTorr, the plasma density exponentially decreases along the axis; the density gradient within $z= \pm 4 \mathrm{~cm}$ is larger due to the effects of the diverging field. For the case of the low pressure $0.35 \mathrm{mTorr}$, the exponential decrease in density is observed only in the upstream side $(z<2 \mathrm{~cm})$ and a slight density dip is observed near $z$ $=2 \mathrm{~cm}$, which indicate an existence of a boundary here. The axial profiles of the plasma potential $\phi_{p}$ measured by the RFEA with the entrance orifice facing radially for 0.35 mTorr (open squares) and 3 mTorr (open circles) are plotted in Fig. 2(b). Here, we mention that the IEDFs in the radial direction have a single peak, which gives the local plasma potential $\phi_{p}$. It is found that a rapid potential drop from $50 \mathrm{~V}$ to $20 \mathrm{Vat} z=-3-1 \mathrm{~cm}$, where the potential gradient is $7-8 \mathrm{~V} / \mathrm{cm}$ and is much larger than the other area (e.g., about $2 \mathrm{~V} / \mathrm{cm}$ at $z<-3 \mathrm{~cm}$ ), is observed for 0.35 mTorr near the outlet of the source tube. This region is just upstream side of the density dip observed in Fig. 2(a) and corresponds to the diverging field area. The plasma potential for 3 mTorr is found to decrease gradually; there is no rapid potential drop. It is considered that the rapid potential drop distinguishes between the upstream and downstream plasmas in the case of low pressure. The recent experiment on the ion acceleration in the geometrically expanding plasma ${ }^{16}$ shows the gradual potential decrease in the diffusion chamber (not in the source tube). In our experiments, the potential drop for the low-pressure case occurs inside the source tube rather than in the diffusion chamber. Moreover, the axial profile of the plasma potential for the case that no magnets are set does not show the rapid potential drop. Hence the potential drop in the present experiments is due to the magnetic-field configuration produced by only the permanent magnets.

Here, it should be discussed whether the potential drop is the DL or the potential gradient following the Boltzmann's relation. The plasma potential $\phi_{p}(z)$ following the Boltzmann's relation is derived as

$$
\phi_{p}(z)=\phi_{p}\left(z_{0}\right)+T_{e} \ln \left[\frac{n_{p}(z)}{n_{p}\left(z_{0}\right)}\right] \text {, }
$$

where $T_{e}$ is the electron temperature $(8 \mathrm{eV}$ for $0.35 \mathrm{mT}$ Torr and $2.5 \mathrm{eV}$ for 3 mTorr) measured by the LP, and $\phi_{p}\left(z_{0}\right)$ and $n_{p}\left(z_{0}\right)$ are the plasma potential and the plasma density at $z$ $=-7 \mathrm{~cm}$, respectively. The plasma potential estimated using Eq. (1) and the results in Fig. 2(a) for 0.35 mTorr and 3 mTorr are plotted in Fig. 2(b) as solid and dotted lines, respectively. It is found that the gradual potential decrease for 3 mTorr is in good agreement with the Boltzmann's relation. For low pressure $0.35 \mathrm{mTorr}$, the estimated potential cannot reproduce the plasma potential profile observed in the experiment; the rapid potential drop is not following the Boltzmann's relation and can be deduced to be the DL.

Moreover, the thickness of the potential drop needs to be addressed here. The recently observed DL in the magnetically expanding plasma has a discontinuous change of the plasma potential and its thickness has been reported to be about $50 \lambda_{\mathrm{De}}{ }^{4}$, , where $\lambda_{\mathrm{De}}$ is the Debye length. On the other hand, there are reports on the formation of the DL with thickness of several centimeters ${ }^{1}$ (several hundreds $\lambda_{\text {De }}$ ) and on the observation of the ion acceleration through $5 \mathrm{~cm}$ thick DL by a laser-induced fluorescence technique. ${ }^{6}$ Our result in Fig. 2(b) shows the potential drop within 3-4 cm (100 $\left.-200 \lambda_{\mathrm{De}}\right)$ from $50 \mathrm{~V}$ to $20 \mathrm{~V}$; its characteristic length is close to the latter DLs. Furthermore, the density dip which is often observed correlating with the DL formation ${ }^{18,19}$ is also formed near the transition area of the plasma potential in our machine. Based on the above-mentioned facts, we suggest the formation of the DL in the plasma expanded by the PMs.

The IEDF measurements in the downstream side are carried out at $z=3 \mathrm{~cm}$ by the RFEA with entrance orifice facing to the source tube; the observed normalized IEDF for 0.35 mTorr and 3 mTorr are plotted in Figs. 3(a) and 3(b) as solid lines, respectively. The IEDF for 0.35 mTorr in Fig. 3(a) clearly shows the existence of the accelerated ions at $V_{c} \simeq 50 \mathrm{~V}$. The beam energy $\varepsilon_{\text {beam }}$ of the accelerated ions can be estimated as about $30 \mathrm{eV}$ because the left-hand peak at $V_{c} \simeq 20 \mathrm{~V}$ shows the local plasma potential. The potential drop at $z=-3-1 \mathrm{~cm}$ can be roughly estimated as $30 \mathrm{~V}$ from the open squares shown in Fig. 2(b). Thus, the beam energy 
(a)

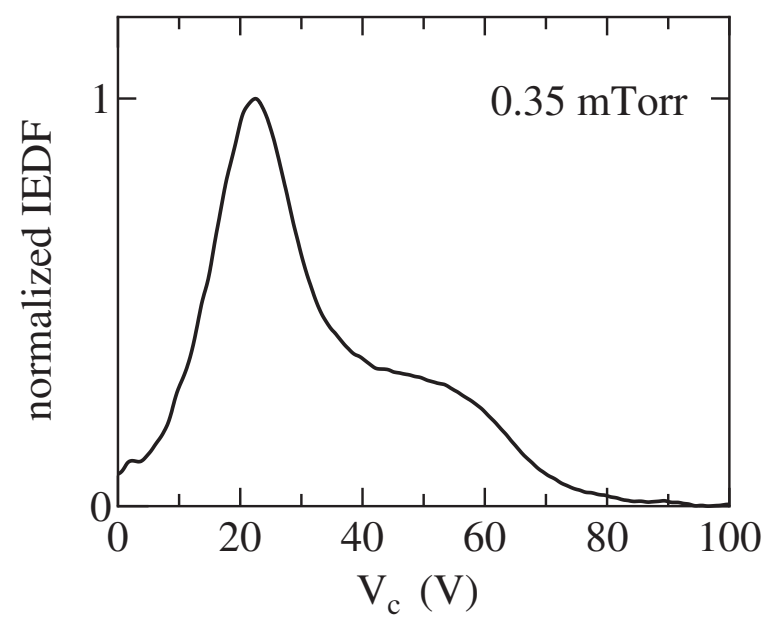

(b)

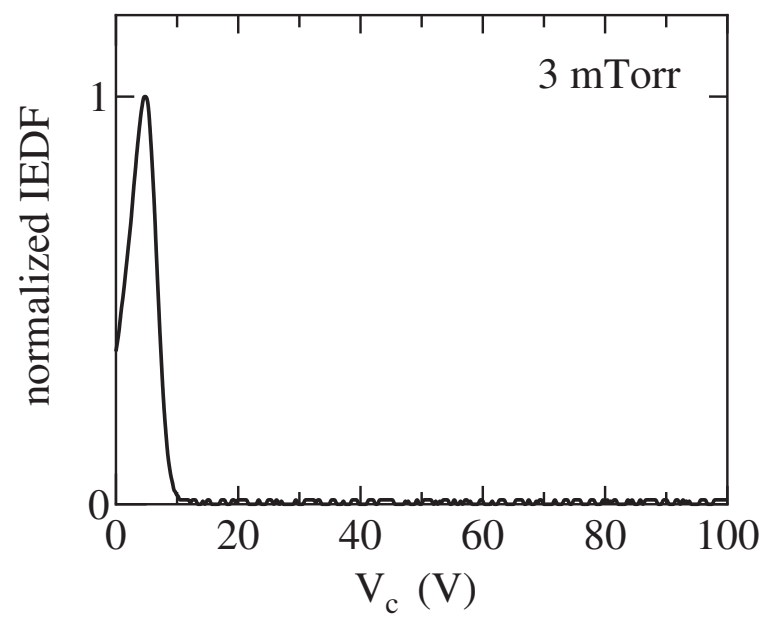

FIG. 3. The IEDFs measured by the RFEA with the entrance orifice facing to the source tube at $z=3 \mathrm{~cm}$ downstream of the rapid potential drop for (a) 0.35 mTorr and (b) 3 mTorr.

is in good agreement with the potential drop. The IEDF for 3 mTorr in Fig. 3(b) has no accelerated ions clearly. These results show evidence of the ion beam formation in the solenoid-free plasma expanded by only the permanent magnets under low pressure conditions. The beam velocity $v_{\text {beam }}$ can be derived as

$$
v_{\text {beam }}=\sqrt{\frac{2 e \varepsilon_{\text {beam }}}{M_{i}}}
$$

where $M_{i}$ is the ion mass. Under the condition of 0.35 mTorr, the beam velocity of ions with $\varepsilon_{\text {beam }} \simeq 30 \mathrm{eV}$ can be estimated as $v_{\text {beam }} \simeq 12 \mathrm{~km} / \mathrm{s}$. The ion sound speed is $C_{s} \simeq 4.4 \mathrm{~km} / \mathrm{s}$ for the electron temperature $T_{e} \simeq 8 \mathrm{eV}$. Thus, the ion beam is found to be supersonic.

The axial profile of the ion beam energy $\varepsilon_{\text {beam }}$ estimated from the IEDF in the axial direction is plotted as closed squares in Fig. 2(b). The beam energy is found to gradually increases with a decrease in the plasma potential $\phi_{p}$, which shows evidence that the potential drop accelerates the ions. Here, at $z=-2 \mathrm{~cm}$, it was difficult to determine the difference between the plasma and the beam potentials because the two peaks are much too close. In addition, we performed the measurement of the ion beam energy at $z=3 \mathrm{~cm}$ and the axial profile of the plasma potential depending on the gas pressure. According to the results, both the beam energy and the po- tential drop decreases with the increase in the gas pressure and suddenly disappear at about $2 \mathrm{mTorr}$. It is observed that the maximum of the beam energy is about $45 \mathrm{eV}$ at the lowest pressure of about 0.3 mTorr.

In summary, we demonstrated the ion beam formation in the solenoid-free plasma expanded by only the permanent magnets, where the cusp field preventing the particle diffusion and the formation of the potential drop are not formed near the outlet of the source tube. The double concentric arrays of the permanent magnet bars located at the different axial positions can produce the constant field area for the plasma production and the diverging magnetic-field area for the potential-drop formation. The potential drop is generated at the diverging field area and can accelerate the ions; we suppose this structure is an electric double layer. The results would be useful for the development of an efficient electric thruster without electromagnets causing the increase in consumed electricity, weight, and cost, although the optimization is needed now. The rf-driven plasmas under the magnetic field would transit to the high-density helicon mode with an increase in rf power under an optimized field configuration, which should be realized with keeping the ion beam formation and is utilized for producing a large amount of thrust. The optimization of the source for the electric thruster is our next challenge.

The authors are indebted to Professor R. Hatakeyama, H. Ishida, Professor T. Kaneko, and M. Kobayashi of Tohoku University for their assistance for constructing the vacuum chamber. This work was supported by a Grant-in-Aid for Young Scientists (B, 20740317) from the Ministry of Education, Culture, Sports, Science and Technology, Japan. This work is also partially supported by the TEPCO Research Foundation.

${ }^{1}$ G. Hairapetian and R. L. Stenzel, Phys. Rev. Lett. 61, 1607 (1988).

${ }^{2}$ S. A. Cohen, N. S. Siefert, S. Stange, R. F. Boivin, E. E. Scime, and F. M. Levinton, Phys. Plasmas 10, 2593 (2003).

${ }^{3}$ W. M. Manheimer and R. F. Fernsler, IEEE Trans. Plasma Sci. 29, 75 (2001).

${ }^{4}$ C. Charles and R. W. Boswell, Appl. Phys. Lett. 82, 1356 (2003).

${ }^{5}$ C. Charles and R. W. Boswell, Phys. Plasmas 11, 1706 (2004).

${ }^{6}$ X. Sun, A. M. Keesee, C. Biloiu, E. E. Scime, A. Meige, C. Charles, and R. W. Boswell, Phys. Rev. Lett. 95, 025004 (2005).

${ }^{7}$ C. Charles and R. W. Boswell, Appl. Phys. Lett. 91, 201505 (2007).

${ }^{8}$ A. Fruchtman, Phys. Rev. Lett. 96, 065002 (2006).

${ }^{9}$ N. Plihon, P. Chabert, and C. S. Corr, Phys. Plasmas 14, 013506 (2007).

${ }^{10}$ C. Charles, Plasma Sources Sci. Technol. 16, R1 (2007).

${ }^{11}$ K. Takahashi, C. Charles, R. W. Boswell, T. Kaneko, and R. Hatakeyama, Phys. Plasmas 14, 114503 (2007).

${ }^{12}$ I. S. Hong, Y. S. Hwang, G. H. Lee, D. Y. Kim, H. Y. Won, G. S. Eom, and W. Choe, Rev. Sci. Instrum. 71, 1385 (2000).

${ }^{13}$ K. Sasaki, H. Kokubu, D. Hayashi, and K. Kadota, Thin Solid Films 386, 243 (2001).

${ }^{14}$ F. F. Chen and H. Torreblanca, Plasma Phys. Controlled Fusion 49, A81 (2007).

${ }^{15}$ K. P. Shamrai, Y. V. Virko, V. F. Virko, and A. I. Yakimenko, in Proceedings of the 42nd AIAA/ASME/SAE/ASEE Joint Propulsion Conference and Exhibit, California, 2006 (AIAA, New York, 2006), p. 4845.

${ }^{16}$ C. S. Corr, J. Zanger, R. W. Boswell, and C. Charles, Appl. Phys. Lett. 91, 241501 (2007).

${ }^{17}$ K. F. Schoenberg, Rev. Sci. Instrum. 49, 1377 (1978).

${ }^{18}$ P. Leung, A. Y. Wong, and B. H. Quon, Phys. Fluids 23, 992 (1980).

${ }^{19}$ R. Schrittwieser, I. Axnas, T. Carpenter, and S. Torven, IEEE Trans. Plasma Sci. 20, 607 (1992). 